\title{
\begin{tabular}{ll} 
Research Square & Preprints are preliminary reports that have not undergone peer review. \\
\hline & They should not be considered conclusive, used to inform clinical practice,
\end{tabular} or referenced by the media as validated information.
}

\section{Sustainability of Yam Daabo Interventions' Effects on Contraception use in Burkina Faso 12 Months after the end of Interventions}

\section{Abou Coulibaly ( $\sim$ samsoncoul@gmail.com )}

Unité de Surveillance Démographique et de Santé (Kaya-HDSS), Institut de Recherche en Sciences de la Santé (IRSS)

\section{Adama Baguiya}

Unité de Surveillance Démographique et de Santé (Kaya-HDSS), Institut de Recherche en Sciences de la

Santé (IRSS)

\section{Franck Garanet}

Unité de Surveillance Démographique et de Santé (Kaya-HDSS), Institut de Recherche en Sciences de la Santé (IRSS)

\section{Nguyen Tran}

UNDP-UNFPA-UNICEF-WHO-World Bank Special Programme of Research, Development and Research

Training in Human Reproduction (HRP), World Health Organization

\section{Tiéba Millogo}

Institut Africain de la Santé Publique

Wambi Maurice Evariste Yaméogo

Institut Africain de la Santé Publique

Ivlabèhire Bertrand Meda

Institut de Recherche en Sciences de la Santé (IRSS)

Blandine Thiéba

Unité de formation et de recherche en sciences de la santé, Université Joseph KI-ZERBO

\section{Séni Kouanda}

Institut de Recherche en Sciences de la Santé (IRSS)

\section{Research Article}

Keywords: family planning, interventions, use, sustainability, impact, postpartum

Posted Date: December 28th, 2020

DOI: https://doi.org/10.21203/rs.3.rs-113062/v1 
License: (c) (i) This work is licensed under a Creative Commons Attribution 4.0 International License. Read Full License 


\section{Abstract}

\section{Introduction}

After testing the interventions for improving the prevalence of contraceptive use, very few studies have measured the sustainability of the effects achieved during implementation. This study aimed to measure the sustainability of Yam Daabo interventions' effects on contraceptive use in Burkina Faso twelve months after these interventions were completed.

\section{Methods}

Yam Daabo was a two-group, multi-intervention, single-blind, cluster randomized controlled trial. Interventions comprised refresher training for the provider, a counseling tool, supportive supervision, availability of contraceptive services 7 days a week, client appointment cards, and invitation letters for partners. We used generalized linear mixed-effects models (log Poisson) to compare the modern contraceptive prevalence at 24 months in the intervention and control groups. We collected data between September and November 2018. We conducted an intention-to-treat analysis and adjusted the prevalence ratios on cluster effects and unbalanced baseline characteristics.

\section{Results}

Twelve months after the completion of the Yam Daabo trial, we interviewed 84.9\% (485 out of 571 women) of the women included at Yam Daabo starting, that is, 247/286 in the intervention group (86.4\%) and $238 / 285$ in the control group (83.5\%). No difference was observed in the use of hormonal contraceptive methods between the intervention and control groups (adjusted prevalence ratio $=1.21 ; 95 \%$ confidence interval $[\mathrm{Cl}]=[0.91-1.61], p=0.191)$. By contrast, women in the intervention group were more likely to use long acting reversible contraceptives (LARC) than those in the control group (adjusted prevalence ratio $=1.35 ; 95 \% \mathrm{Cl}=[1.08-1.69], \mathrm{p}=0.008)$.

\section{Conclusion}

This study showed that women in the intervention group preferred to use long-acting reversible contraceptives even though no significant difference was found in the comparison of modern contraceptive prevalences between the two arms.

\section{Trial registration}

The trial registration number at the Pan African Clinical Trials Registry is PACTR201609001784334 and the date of the first registration is $27 / 09 / 2016$.

\section{Introduction}


Many authors have tested several interventions to improve the prevalence of contraceptive use [1, 2]. In 2015, we tested a package of interventions in two African countries, Burkina Faso (BF) and the Democratic Republic of the Congo (DRC), in a randomized cluster trial design called Yam Daabo [3, 4]. The PPFP intervention package had two types of interventions. The first one comprised three facilityoriented interventions: refresher training of service providers, regularly scheduled and strengthened supportive supervision of providers, enhanced availability of services 7 days a week. The second had three individual-based interventions: a PPFP counseling tool, appointment cards for women, and invitation letters for partners. The conception of the new counseling tool takes into account all new World Health Organization [WHO] recommendations on family planning service provision. The package was designed through participatory action research, and the process and contents are detailed elsewhere [4]. After 12 months of follow-up (which marks the end of the interventions), the main results showed a significant increase in the use of appropriate modern contraceptive methods among women receiving the interventions compared to women not receiving the interventions. In BF, at 12 months, the modern contraceptive prevalence rate was 55\% among women in the intervention group and $29 \%$ among women in the control group (adjusted prevalence ratio [PR]: 1.79, 95\% Cl $=1.30-2.47$ ) [5]. In the DRC, at 12 months, $46 \%$ of the women in the intervention group and $35 \%$ of the women in the control group were using modern contraceptives (adjusted PR: $1.58,95 \% \mathrm{Cl}=0.74-3.38$ ), with significant differences in the use of contraceptive implants (22\% vs. $6 \%$; adjusted PR: $4.36,95 \% \mathrm{Cl}=1.96-9.70)$ [6].

However, many questions remain unanswered a few months after these types of interventions because most studies examine the effects of interventions immediately after the interventions' completion. So, very few studies have measured the sustainability of the effects of interventions that were effective after they were discontinued. A systematic review conducted in 2016 by Iwelunmor et al. [7], found studies on infectious diseases (human immunodeficiency virus/acquired immunodeficiency syndrome and malaria). However, a few studies measured the effects of family planning interventions a few months or years after they were discontinued in Nigeria (involving community-based interventions) [8-10].

As three (out of six) of Yam Daabo's interventions were individual-based, we hypothesized that (i) health workers could continue to offer some elements of the intervention package to women visiting the health centers, (ii) the effects may persist even after the interventions were stopped as women will receive counseling several times (during and after the first year postpartum), (iii) women in the intervention group not using the methods at the end of the interventions because of past and current exposures may start using contraceptive methods one year after giving birth. We hypothesize that appropriate contraceptive methods use would remain high within 24 months among women in the intervention group compared to those in the control group. Therefore, this study aimed to measure the effects of the Yam Daabo trial interventions on contraceptive methods use in BF, 12 months after the end of the interventions, corresponding to 24 months postpartum.

\section{Methods}


We conducted a complex, multicentre, randomized cluster trial called Yam Daabo in primary health centers in the Yako health district (Centre-North region, BF).

$\mathrm{BF}$ is a sub-Saharan African country, with an estimated population of 20,321,000 in 2019 [11]. According to Blumenberg et al., the modern contraceptive prevalence rate (MCPR) was 31.9\% in December 2018, based on the analysis of Performance Monitoring and Accountability 2020 (PMA2020) data [12]. Ahmed et al., using the FP 2020 data, reported an estimated unmet need for modern contraception of $27.2 \%$ in 2017 [13]. As in most countries in sub-Saharan Africa, cultural norms favor large families, with higher fertility rates in rural areas than in urban areas. In 2019, BF had 5.6 live births per woman [11].

\section{Description of the Yam Daabo trial:}

This study involved two countries: BF and the DRC. The project was implemented in several successive phases: pre-formative phase, formative phase, and intervention phase. We randomized each country's health centers into two groups during the intervention phase: intervention and control. The health centers in the experimental group offered six PPFP interventions that were identified as solutions to the barriers identified during the formative phase of the project and are already described in another article [4]. The health centers in the control group offered the usual PPFP care. We included and followed women at the third trimester of their pregnancy over 12 months after delivery. As said above, Yam Daabo's interventions comprised refresher training for the provider, counseling tool, supportive supervision, availability of contraceptive services 7 days a week, client appointment cards, and invitation letters for partners. Participants received individual-based interventions during their pregnancy and during the postpartum period, according to national practice (typically on clinical discharge [24-48 h], at 6 days, 6 weeks, then at months 6 and 9, before the trial exit at month 12 postpartum). The study was statistically powered to detect a difference of 15 percentage points between the experimental and control groups in the proportion of women adopting an effective method of PPFP at 6 months postpartum. Considering the intra-cluster correlation coefficient, in each country, each study group had to have 4 health centers with at least 60 participants per centre to have a statistical power of $93 \%$ at a significance level of $5 \%$. As this study had an estimated loss to follow-up rate of $10 \%$, each center had to recruit at least 70 pregnant women. This value was equivalent to a cohort of 280 pregnant women in each group ( 4 centers $\times 70$ participants) and a total of 560 participants per study and country ( 280 participants $\times 2$ study groups). All pregnant women were eligible to participate in the study if (1) they were in their third pregnancy trimester; (2) the status of the pregnancy and the woman allowed for a birth at the health center; (3) the woman had the intention to attend ANC, delivery, and PNC at the health center; (4) the woman did not participate in another study; and (5) we obtained informed consent. Research assistants collected data on paperbased case report forms (CRFs). The WHO team in Geneva developed the CRFs with inputs from the country's research teams. Each health center of the cluster RCT had a research assistant who was trained to adhere to the study manual and standard operating procedures for data management, which are common to both study countries and have been developed by WHO. The information reported on the CRFs was checked for accuracy and completeness several times at different levels by field coordinators 
and data managers. The Biostatistics and Data Management team at HRP developed the eCRF through OpenClinica (version 3.11), an electronic data capture software for clinical research. This software allowed checks for data accuracy, completeness, and consistency, reducing data queries, and problem resolution delays. In each country, the eight sites were matched by pairs according (1) the average number of deliveries per month, (2) the ratio of health workers per population, and (3) the settings (rural, urban). Within each pair, we randomly selected the site assigned to the experimental intervention. This randomization was done four times. No restriction in the randomization process was required. All consecutive and eligible participants were included in the clusters. Due to the nature of the interventions, participants, health staff, research assistants assigned to each centre, and the rest of the research team members could not be masked to the cluster assignments. Methodological details on the Yam Daabo are available in the published protocol [3].

The trial was approved by the WHO Ethics Committee and the Health Research Ethics Committee of BF and has been registered in the Pan-African Clinical Trials Registry (registration number:

PACTR201609001784334 on 27/09/2016).

No interim analysis was conducted during this study. At the end of the 12-month follow-up, analyses on contraceptive use at each of the contact points (day 6 , week 6 , month 6 , and month 12) were conducted. These results for BF and the DRC have already been published $[5,6]$.

The time from enrolment to exit interview at 24 months postpartum spanned from July 27, 2016, to November 15, 2018.

\section{Type of study}

We conducted a randomized, clustered, controlled trial extending the follow-up of women to 24 months (12 months after the end of the interventions).

\section{Population}

we included in this study all postpartum women who participated in the Yam Daabo trial.

\section{Additional collection period}

we collected data from September 15, 2018, to November 15, 2018.

\section{Key measures}

The primary outcome of this study was the contraceptive method use. We used the classification adopted by the WHO in 2015 [14]. Thus, for this analysis, the groups of methods were defined as follows 
- Long-acting reversible contraceptive, including implants and intrauterine devices

- Short-acting contraceptive methods, including injectables, pills, emergency contraception, male and female condoms

- Permanent methods (male and female sterilization)

- The lactational amenorrhea method

Next, we also classified the contraceptive methods into "modern and appropriate methods" and "nonmodern or inappropriate methods." The non-modern methods comprised traditional methods, withdrawal, and abstinence. Inappropriate methods include lactational amenorrhea (if used after 6 months) and calendar-based methods (if used during the first 12 months postpartum).

The main dependent variable was exposure to interventions, defined as the binary variable (coded 1 for the intervention group and 0 for the control group).

Moreover, we measured the frequency of pregnancies in both groups of women.

\section{Data collection at 24 months postpartum}

five interviewers collected the data (They were part of the interviewing team that collected follow-up data up to 12 months postpartum). To locate the target participants, they used the women's identification documents, which contained their telephone numbers. Maternity health workers and community-based health workers provided valuable assistance in the search for women. They conducted interviews mainly in health centers and women's homes or other secure public places chosen by the woman beforehand. Data on contraception use were extracted into the health centers registers.

\section{Data processing and statistical analysis}

EpiData and Stata 15.1 software were used for data entry and analysis, respectively. To ensure the comparability of results with published data on intervention effectiveness at 12 months postpartum, we used generalized linear mixed-effects (log Poisson) models were used to measure the effects of interventions on contraceptive use with a significance threshold of $5 \%$. We adjusted the measure of association on sociodemographic variables unequally distributed between the intervention and control 
groups, at inclusion. We also corrected for a possible cluster effect by taking into account any correlation that might exist.

We followed Consolidated Standards of Reporting Trials extension for pragmatic trials guidelines to write the manuscript (supplementary file 1).

\section{Ethical considerations}

The study protocol was approved by the Health Research Ethics Committee of BF. Participants' data were anonymized, and informed consent was obtained from all women prior to data collection.

\section{Results}

Twelve months after the end of the Yam Daabo interventions, we found that 485 (87.4\%) of 555 women used contraceptive methods. The lost to follow-up rates were $13.6 \%$ in the intervention group (247 of 286 women included) and $16.5 \%$ in the control group (238 of 285 women included). The flow diagram, presented in Fig. 1, summarizes this information. However, no statistical difference was observed in the lost to follow-up rates between the two groups $(p=0.332)$.

The chi-square test comparing women's sociodemographic and gynecological characteristics at baseline showed no significant difference in any of the variables except the total number of abortions $(p=0.01)$. These data are presented in Table 1. 
Table 1

baseline characteristics of women who were interviewed at 24-month postpartum (inclusion data)

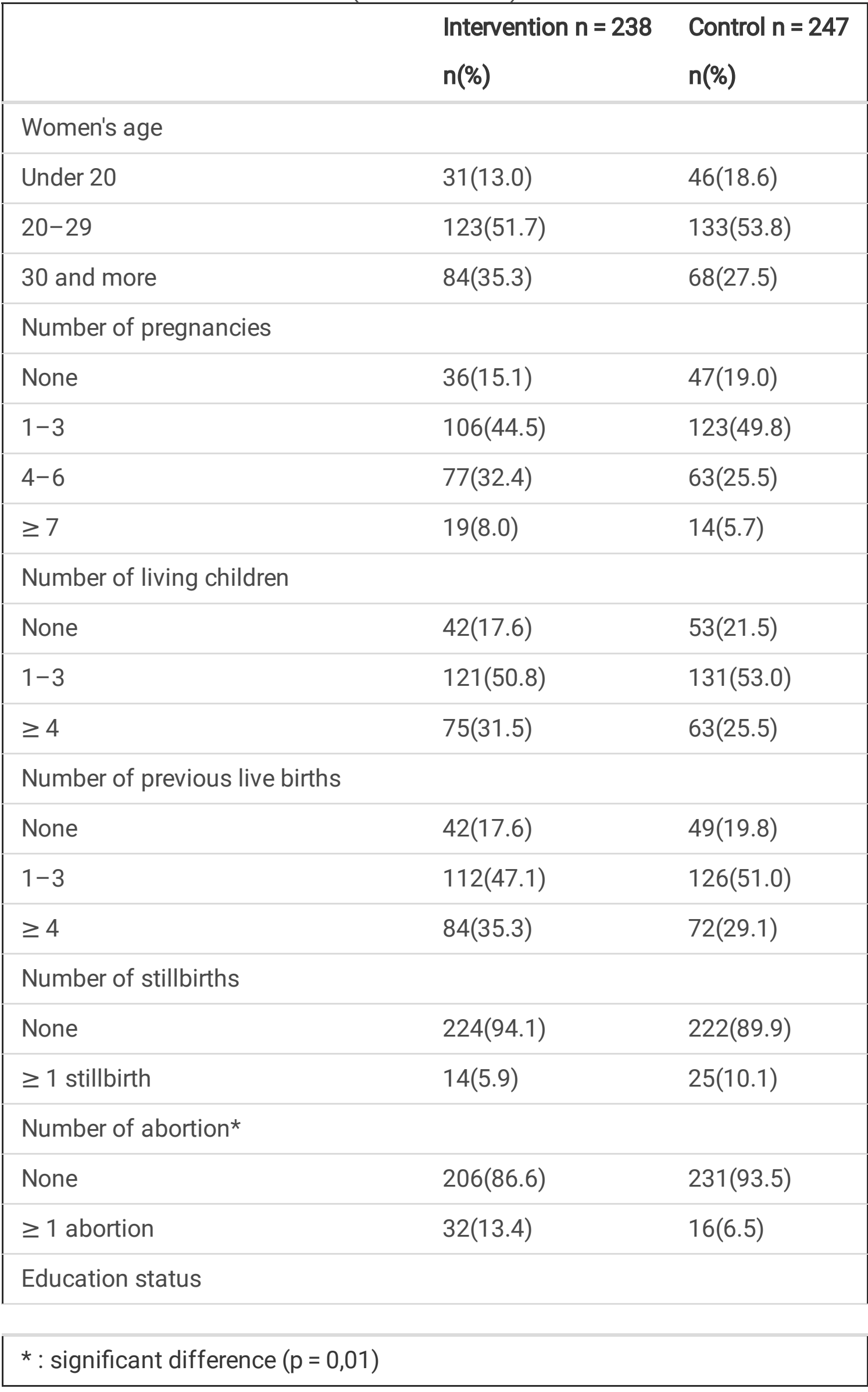




\begin{tabular}{|lll|}
\hline & $\begin{array}{l}\text { Intervention } \mathbf{n = 2 3 8} \\
\mathbf{n}(\%)\end{array}$ & $\begin{array}{l}\text { Control } \mathbf{n}=\mathbf{2 4 7} \\
\mathbf{n}(\%)\end{array}$ \\
\hline No education & $163(68.5)$ & $174(70.4)$ \\
\hline Primary school & $35(14.7)$ & $36(14.6)$ \\
\hline Secondary/Tertiary & $40(16.8)$ & $37(15.0)$ \\
\hline Marital status & & \\
\hline Not in union & $2(0.8)$ & $4(1.6)$ \\
\hline In union & $236(99.2)$ & $243(98.4)$ \\
\hline Occupation & & $0(0.0)$ \\
\hline No occupation & $1(0.4)$ & $19(7.7)$ \\
\hline Student & $24(10.1)$ & $201(81.4)$ \\
\hline Housewife/Farmer & $185(77.7)$ & $5(2.0)$ \\
\hline Salaried employee & $2(0.8)$ & $22(8.9)$ \\
\hline Tradeswoman & $26(10.9)$ & $113(45.7)$ \\
\hline Family planning use prior to the trial & & $178(72.1)$ \\
\hline No & $161(67.6)$ & $69(27.9)$ \\
\hline Yes & $77(32.4)$ & \\
\hline Current pregnancy planned & & \\
\hline Yes & $115(48.3)$ & \\
\hline No & $123(51.7)$ & \\
\hline * significant difference $(\mathrm{p}=0,01)$ & & \\
\hline
\end{tabular}

Results showed that women in the intervention group had a higher prevalence of modern contraceptive use than those in the control group at 24 months postpartum (58.0\% vs. $47.4 \%$; Adjusted PR $=1.21 ; 95 \%$ $\mathrm{Cl}=0.91-1.61, \mathrm{p}=0.191)$, but this difference was not statistically significant. The use of LARC was higher among women in the intervention group than in those in the control group with a statistically significant difference (37.4\% vs. $27.5 \%$; Adjusted $\mathrm{PR}=1.35 ; 95 \% \mathrm{Cl}=1.08-1.69, \mathrm{p}=0.008)$. No significant difference was noted in the use of short-acting methods ( $20.6 \%$ vs. $19.8 \%$; Adjusted PR $=1.00,95 \% \mathrm{Cl}=$ $0.55-1.81, p=0.992$ ). Among the non-modern or inappropriate methods, only one woman was using the standard days' method. None of the women used a permanent method (vasectomy or tubal ligation) at 24 months postpartum. Table 2 expressed these results. 
Table 2

contraception use at 24-month

\begin{tabular}{|lllll|}
\hline & $\begin{array}{l}\text { Intervention } \\
\text { group } \\
\mathrm{n} / \mathrm{N}\end{array}$ & $\begin{array}{l}\text { Control } \\
\text { group } \\
\mathrm{n} / \mathrm{N}\end{array}$ & $\mathbf{p}$ & $\begin{array}{l}\text { Adjusted prevalence } \\
\text { ratio (95\%Cl) }\end{array}$ \\
\hline $\begin{array}{l}\text { Modern and appropriate } \\
\text { methods }\end{array}$ & $\begin{array}{l}138 / 238 \\
(58.0 \%)\end{array}$ & $\begin{array}{l}117 / 247 \\
(47.4 \%)\end{array}$ & 0.191 & $1.21(0.91-1.61)$ \\
\hline Long-acting methods & $\begin{array}{l}89 / 238 \\
(37.4 \%)\end{array}$ & $\begin{array}{l}68 / 247 \\
(27.5 \%)\end{array}$ & 0.007 & $1.35(1.08-1.69)$ \\
\hline Short-acting methods & $\begin{array}{l}49 / 238 \\
(20.6 \%)\end{array}$ & $\begin{array}{l}49 / 247 \\
(19.8 \%)\end{array}$ & 0.992 & $1.00(0.55-1.81)$ \\
\hline $\begin{array}{l}\text { Non-modern or non-appropriate } \\
\text { methods }\end{array}$ & $1 / 238(0.4 \%)$ & $0 / 247(0 \%)$ & - & - \\
\hline No contraceptive method & $\begin{array}{l}100 / 238 \\
(42 \%)\end{array}$ & $\begin{array}{l}130 / 247 \\
(53 \%)\end{array}$ & 0.224 & $0.8(0.56-1.15)$ \\
\hline
\end{tabular}

Concerning the frequency of pregnancies, we noted no significant difference between women in the intervention and control groups (21 out of 238 pregnant women in the intervention group versus 23 out of 247 in the control group, $8.8 \%$ and $9.3 \%$, respectively).

\section{Discussion}

This analysis has two main findings. First, Yam Daabo trial interventions' effects on modern family planning use persisted 12 months after the interventions ended, but no statistically significant difference was noted between the experimental and control groups. Second, a statistically significant difference was noted in its effects on LARC use.

Some authors, including Tu et al. [15], Speizer et al. [8, 9], Subramanian et al. [16], and Jejeebhoy et al. [17], after evaluating the sustainability of the effects of certain interventions related to contraceptives' provision several months after the cessation of interventions noted that the results varied in terms of the intensity of the sustainability of the effects. Indeed, in Shanghai, Tu et al. noted a significant difference in contraceptive method use and reported a higher contraceptive use in the group of young people who benefited from the interventions 28 months after the cessation of interventions [15]. Subramanian et al. [16] and Jejeebhoy et al. [17] reported the persistence of effects 4 to 8 years after the end of the PRACHAR project, implemented between 2001 and 2012 in Bihar (India), on contraceptive use among young married couples. Speizer et al. noted the persistent effects of a community-based program on contraceptive use in two cities where the program had been implemented [8] and a change in the effects of the same program on the quality of family planning services after the program ended [9]. The interventions' nature can explain Yam Daabo interventions sustainability effects (significant difference in the use of LARC) with three individual-based rather than community-based. One could be led to believe that the skills women learned during the implementation phase of the interventions guided them for 
12 months after the interventions' completion. This is a cause for satisfaction because the cessation of the interventions coincided with the end of repeated contact period between postpartum women and health workers. In Burkina Faso's context, these contacts are limited to 12 months postpartum.

The non-significant difference at 24-month in modern contraceptive use between the experimental and control groups could be explained by the effects of two campaigns of free contraceptive methods' distribution since the last follow-up. These two campaigns were conducted in all public health facilities (7 days each, including one campaign in November and another one in June each year). Compared with the results published at 12 months, this non-significant difference in the modern contraceptive prevalence can be explained by the increased contraceptive use in the control group ( $29 \%$ at 12 months [5] vs. $47.4 \%$ at 24 months). This net increase in the control group could be related to the effects of the national family planning week, which is celebrated every June and November and covers all regions of BF, during which contraceptives are provided free of charge. Such a campaign for free contraceptive methods could therefore have more effect in the control group (which included women who used the methods less) than in the intervention group since there was a $55 \%$ use rate at 12 months [5]. It could be argued that because of the higher proportion of unmet needs in the control group, it is easier to register new users as soon as free distribution campaigns take place. Current data on the modern contraceptive prevalence in the general population could help us better comprehend this rate's evolution in the control group. Thus, if contraceptive methods were free, women would use more modern methods. In the Burkinabe context, the implementation of the interventions tested in this study, combined with the free distribution of contraceptive products (already ongoing in Burkina since 2019 in some regions and since 2020 throughout the country), could allow the widespread use of modern contraceptive methods.

Our study had several limitations. First, the proportion of people who were lost to follow-up was slightly high. However, this did not affect our results' internal validity in terms of power since the initial hypothesis was that 60 women per health center, 480 women in total, should be included to achieve the minimum desired power [3]. However, we selected and surveyed 485 women. Another limitation was that we had no contact with the women 12 months after the study. As a result, the participants may have forgotten several episodes.

\section{Conclusion}

This study showed that the effects of interventions in improving contraceptive use can be sustained for up to 12 months after the interventions are completed. Indeed, women continued to use appropriate contraceptive methods and LARC 12 months after the interventions were discontinued. The modern contraceptive prevalence of the intervention group may have important implications in BF. Indeed, if Yam Daabo's realistic interventions are implemented and combined with free contraceptive methods, nearly 3 out of 5 women will still be using modern contraceptive methods at 24 months. This result is more significant as contraceptive methods have been provided free of charge in BF's public health facilities since 2020 . 


\section{Declarations}

\section{Ethics approval and consent to participate}

The study protocol was approved by the Health Research Ethics Committee of Burkina Faso. Participants' data were anonymized, and informed consent was obtained from all women prior to the 24 months postpartum data collection. It has been registered in the Pan-African Clinical Trials Registry (registration number: PACTR201609001784334, registered on 27/09/2016).

\section{Statement on the inclusion of minor participants}

In the case of minor participants, informed consent of the parent or the respective legal guardian was obtained in conjunction with informed assent of the underage participant (less than 18 years of age).

\section{Statement on all the procedures in this research}

All methods were carried out in accordance with relevant guidelines and regulations.

\section{Consent for publication}

Not applicable

\section{Availability of data and materials}

The datasets used and/or analysed during the current study are available from the corresponding author on reasonable request.

\section{Competing interests}

The authors declare that they have no competing interests

\section{Funding}

The Government of France funded Yam Daabo through the Muskoka Funds and the 24-month data collection was carried out with the World Health Organization's financial support. The study funder (Government of France or World Health Organization) had no role in study design, data collection, data analysis, data interpretation, or writing this article. 


\section{Authors' contributions}

AC participated in all phases of the project: from design to data collection. He analyzed the data for this article and wrote the first draft with the important contributions from AB, FG, TM, NTT, BM, WMEY, BT, and SK. TM, NTT, MWEY, BT and SK were members of Yam Daabo's research team. SK was the principal investigator of the project in $\mathrm{BF}$ and is the supervisor for this thesis. All authors contributed to revising the paper and agreed to be accountable for all aspects of the work.

\section{Acknowledgments}

This work was carried out as part of the epidemiology thesis of the corresponding author. Special thanks go to the World Health Organization for offering the scholarship of doctoral studies in epidemiology. We also thank the biomedical and public health department of the Research Institute of health sciences.

\section{References}

1. Blazer C, Prata N. Postpartum family planning: current evidence on successful interventions. Open Access J Contracept. 2016;7:53-67.

2. Cleland J, Shah IH, Daniele M. Interventions to Improve Postpartum Family Planning in Low-and Middle-Income Countries: Program Implications and Research Priorities. Stud Fam Plann. 2015;46:423-441.

3. Tran NT, Gaffield ME, Seuc A, Landoulsi S, Yamaego WME, Cuzin-Kihl A, et al. Effectiveness of a package of postpartum family planning interventions on the uptake of contraceptive methods until twelve months postpartum in Burkina Faso and the Democratic Republic of Congo: the YAM DAABO study protocol. BMC Health Serv Res. 2018;18:439.

4. Tran NT, Yameogo WME, Langwana F, Gaffield ME, Seuc A, Cuzin-Kihl A, et al. Participatory action research to identify a package of interventions to promote postpartum family planning in Burkina Faso and the Democratic Republic of Congo. BMC Womens Health. 2018;18:122.

5. Tran NT, Seuc A, Coulibaly A, Landoulsi S, Millogo T, Sissoko F, et al. Post-partum family planning in Burkina Faso (Yam Daabo): a two group, multi-intervention, single-blinded, cluster-randomised controlled trial. Lancet Glob Health. 2019;7:e1109-17.

6. Tran NT, Seuc A, Tshikaya B, Mutuale M, Landoulsi S, Kini B, et al. Effectiveness of postpartum family planning interventions on contraceptive use and method mix at 1 year after childbirth in Kinshasa, DR Congo (Yam Daabo): a single-blind, cluster-randomised controlled trial. Lancet Glob Health. 2020;8:e399-410.

7. Iwelunmor J, Blackstone S, Veira D, Nwaozuru U, Airhihenbuwa C, Munodawafa D, et al. Toward the sustainability of health interventions implemented in sub-Saharan Africa: a systematic review and conceptual framework. Implement Sci. 2016;11:43. 
8. Speizer IS, Guilkey DK, Escamilla V, Lance PM, Calhoun LM, Ojogun OT, et al. On the sustainability of a family planning program in Nigeria when funding ends. PLOS ONE. 2019;14:e0222790.

9. Speizer IS, Calhoun LM, McGuire C, Lance PM, Heller C, Guilkey DK. Assessing the sustainability of the Nigerian urban reproductive health initiative facility-level programming: longitudinal analysis of service quality. BMC Health Serv Res. 2019;19:559.

10. Scheirer MA, Dearing JW. An Agenda for Research on the Sustainability of Public Health Programs. Am J Public Health. 2011;101:2059-67.

11. United Nations. Country profile: Burkina Faso. UNdata website. http://data.un.org/en/iso/bf.html. Accessed October 192020.

12. Blumenberg C, Hellwig F, Ewerling F, Barros AJD. Socio-demographic and economic inequalities in modern contraception in 11 low- and middle-income countries: an analysis of the PMA2020 surveys. Reprod Health. 2020;17:82.

13. Ahmed S, Choi Y, Rimon JG, Alzouma S, Gichangi P, Guiella G, et al. Trends in contraceptive prevalence rates in sub-Saharan Africa since the 2012 London Summit on Family Planning: results from repeated cross-sectional surveys. Lancet Glob Health. 2019;7:e904-11.

14. World Health Organization. Medical eligibility criteria for contraceptive use. Fifth edition. 2015.

15. Tu X, Lou C, Gao E, Shah IH. Long-Term Effects of a Community-Based Program on Contraceptive Use Among Sexually Active Unmarried Youth in Shanghai, China. J Adolesc Health. 2008;42:249-58.

16. Subramanian L, Simon C, Daniel EE. Increasing Contraceptive Use Among Young Married Couples in Bihar, India: Evidence From a Decade of Implementation of the PRACHAR Project. Glob Health Sci Pract. 2018;6:330-44.

17. Jejeebhoy SJ, Prakash R, Acharya R, Singh SK, Daniel E. Meeting Contraceptive Needs: Long-Term Associations Of the PRACHAR Project with Married Women's Awareness and Behavior in Bihar. Int Perspect Sex Reprod Health. 2015;41:115-25.

\section{Figures}




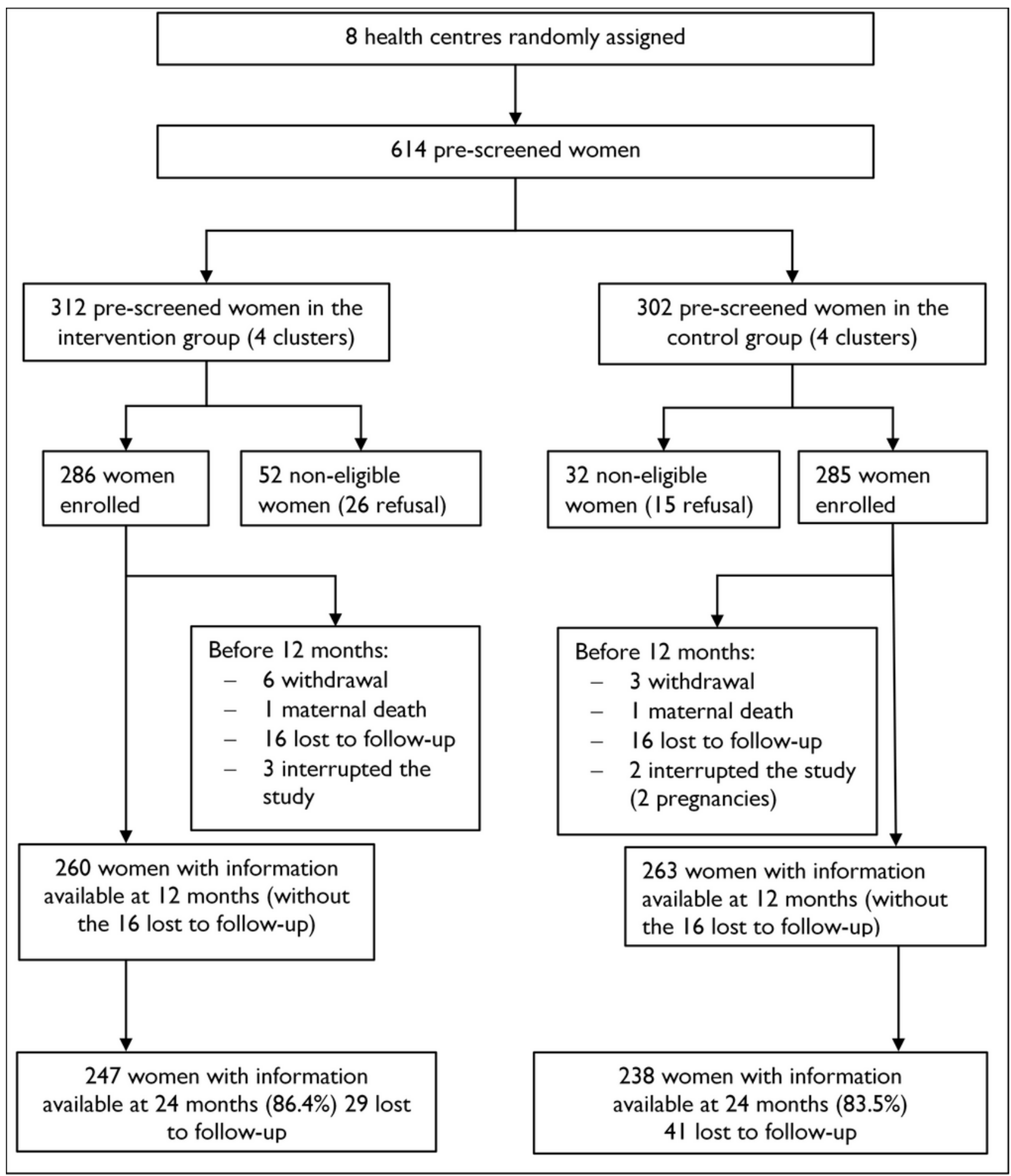

Figure 1

flow diagram

\section{Supplementary Files}

This is a list of supplementary files associated with this preprint. Click to download. 
- SustainabilityTables18Nov2020.docx 